\title{
EL MODELO DE ENSEÑANZA-APRENDIZAJE DE LAS CIENCIAS EN LA REFORMA DE LAS ENSEÑANZAS MEDIAS
}

Barandiaran piedra, J. Instituto de Formación Profesional Castrillón (Asturias).

\section{SUMMARY}

In this paper the study of theoretical basis and educational assumptions of the proposals for experimental science contained in the "Project for the reform of the spanish educational system» is faced.

\section{INTRODUCCIÓN}

El pasado mes de junio el Ministerio de Educación y Ciencia presentaba el Proyecto para la Reforma de la Enseñanza, documento que recoge la propuesta oficial para una nueva organización del sistema educativo, que incluirá, entre otros cambios, las reformas curricula* res de los distintos niveles de enseñanza no universitaria.

La presentación del proyecto aparece como un paso más en el proceso de reformas educativas -entre las que se encuentra la Reforma de las Enseñanzas Medias - iniciado hace cuatro años y en él se invita a todos los sectores de la sociedad implicados en la educación a debatir las propuestas que contiene.

En estos momentos en los que se avecinan transformaciones profundas del sistema educativo, el presente trabajo aspira a aportar, en la medida de lo posible, algunas ideas y reflexiones sobre una parcela bien definida: la enseñanza de las Ciencias. Ideas y reflexiones fruto, en buena parte, de la experimentación de la Reforma de Enseñanzas Medias como profesora de Fisica y Química durante los dos últimos cursos.

Como es bien sabido, en el curso $83 / 84$ comenzó la fase experimental del proyecto de Reforma de las Enseñanzas Medias (REM) propuesto por el MEC, incorporándose cada curso nuevos centros, profesores y alumnos a la experiencia. Esta Reforma supone, entre otras cosas, la eliminación de la doble vía de escolarización de los alumnos al término de la EGB (los actuales BUP y Formación Profesional de primer grado) y la implantación de un primer ciclo de Enseñanza Media, igual y obligatorio para todos los alumnos: el Bachillerato General. Este primer ciclo consta de dos cursos y los alumnos que los cursan tienen edades comprendidas entre los 14 y los 16 años. Aquellos alumnos que lo supe- ren y lo deseen, pueden seguir al segundo ciclo, cursando cualquiera de los seis Bachilleratos que actual. mente se experimentan.

Después de cuatro años de experimentación del Bachilerato General, y en los momentos actuales -caracterizados por la proximidad de grandes cambios en el sistema educativo como ya he señalado, y muy especialmente en la Enseñanza Media - muchos profesores que hemos participado directa o indirectamente en la experiencia seguimos preocupados por aspectos relacionados con la fundamentación teórica y los presupuestos didácticos del proyecto.

Según el calendario previsto por el MEC para la implantación de la Reforma, durante el curso 1987/88 co. menzará la elaboración del diseño curricular base para todos los niveles educativos, que incluirá los mínimos curriculares comunes a todos los alumnos. Parece lógico esperar que las personas que participen directamente en su elaboración tengan en cuenta la experiencia de estos cuatro antos, incorporando los aspectos positivos de la misma y rechazando o modificando los que se hayan mostrado inadecuados.

Parece oportuno pues, en este contexto, estudiar detenidamente la propuesta del MEC para la Reforma que se ha experimentado estos cuatro años, en lo que se refiere al área de Ciencias Experimentales. He querido centrar el estudio en la fundamentación teórica y los presupuestos didácticos de las propuestas para Ciencias Experimentales, esto es, el modelo de enseñanza aprendizaje de las Ciencias que las orienta.

\subsection{Objetivos}

El modelo de enseñanza-aprendizaje de las Ciencias que respalda las propuestas curriculares no aparece expli- 
citamente en los documentos que las desarrollan, ni siquiera las fuentes que han informado este diseño curricular y los supuestos teóricos asumidos (respecto a la educación escolar, el conocimiento en general y el científico en particular, el aprendizaje, etc.).

El problema consiste, pues, en determinar cuál es el modelo de enseñanza-aprendizaje de las Ciencias que subyace a las propuestas, mediante un análisis de las mismas que permita establecer cuáles son las característi. cas que configuran dicho modelo. Este análisis es lo que constituye la primera parte del trabajo; la segun. da parte es un breve resumen de las implicaciones educativas de la investigación didáctica más reciente, prin. cipalmente de aquellas más relacionadas con el diseño y desarrollo curriculares; por último, en la tercera parte, se procede a un estudio comparativo entre los rasgos más relevantes del modelo de la Reforma y los modelos de enseñanza-aprendizaje de las Ciencias que han surgido en los últimos años fruto de la investigación didáctica, y se señalan los aspectos que deberían astmir propuestas futuras.

\subsection{Los documentos}

Las propuestas iniciales para la REM aparecieron en el llamado «Libro Verde»: Hacia la Reforma, en julio de 1983. A los dos antos de experimentacion, cuando la primera promoción de alumnos terminaba el primer ciclo, se procedió a la revisión del Libro Verde y se publicó en 1985 Hacia la Reforma. Documentos. Continuaron otros documentos que desarrollaban aspectos concretos de la propuestas: los Documentos complementarios I sobre objetivos comunes, interdisciplinaridad y evaluación y los Documentos complementarios $I I$ que continúan desarrollando el tema de la evalua. ción. Además de estos documentos básicos, están los que se han ido recibiendo en los centros experimentales, procedentes unos del Equipo de Apoyo de la Re. forma y otros de los Responsables de Materia de cada área específica. En el Anexo aparece una relación detallada de los que se han ido examinando en este trabajo.

\subsection{El modelo dídáctico}

Como instrumento de análisis del diseno curricular de la Reforma se ha utilizado el Modelo didáctico que Gimeno Sacristán propone en su libro Teoría de la enseñanza y desarrollo del currículo (1981), por ser bastante conocido entre el profesorado y porque cumple las caracteríticas exigibles a un modelo comprensivo de todos los aspectos que un planteamiento científico de la enseñanza debe abarcar: exhaustividad y estructura sistemática.

El autor lo define como una estructura sistemática compuesta por seis elementos básicos: objetivos, contenidos, medios, relaciones de comunicación, organización y evaluación. A su vez, cada uno de estos componentes del modelo posee una seric de dimensiones o aspec- tos relevantes de los mismos, en torno a los cuales cabe la posibilidad de tomar diferentes opciones. Estas opciones son, precisamente, las que configuran la variedad de estrategias didácticas posibles, que se diferencian entre si precisamente porque suponen tomas de posiciones particulares en esas dimensiones de los elementos det modelo didáctico.

Hay que destacar también que las distintas posturas frente a los elementos del modelo que se asumen $\rightarrow$ implícita o explícitamente- suponen una serie de concepciones relacionadas con teorias psicosociologicas del desarrollo y del aprendizaje y están condicionadas por el contexto sociocultural en que el proceso de enseñanza-aprendizaje se desarrolia.

El esquema general que propone Gimeno en su obra ya citada supone, pues: el subsistema netamente didáctico (o modelo didáctico), que se completa con el subsistema psicológico para comprender la dinámica del funcionamiento del propio proceso de enseñanzaaprendizaje, considerando que la interacción de ambos tiene lugar dentro del contexto sociocultural con el que mantiene una serie de relaciones recíprocas.

En este caso, utilizar este modelo didáctico como instrumento de análisis supone determinar las opciones implícitas en las propuestas de la Reforma en torno a las dimensiones de los elementos con mayor potencialidad configuradora (asi por ejemplo, no he incluido en el trabajo el elemento medios por no encontrar apenas ninguna referencia a ellos en los documentos). Estas opciones constituirán las coordenadas que definirán el modelo metodológico de la Reforma. A partir de ellas podrá inferirse las concepciones que las fundamentan, relacionadas tanto con la psicología como con la epistemologia. Por último, se podrá esbozar el modelo de enseñanza.aprendizaje de las Ciencias que orienta las propuestas de la Reforma, señalando sus rasgos más característicos.

\section{EL MODELO DE LA REFORMA WN EL ÁREA DE CIENCIAS EXPERIMENTALES}

Antes de proceder al análisis de los presupuestos en el área de Ciencias Experimentales es conveniente referirse al modelo de la Reforma. No me extenderé demasiado en este punto pues un análisis de las propuestas generales de la Reforma, tanto del Ciclo Superior de EGB como de las Enseñanzas Medias, ya fue acometido con anterioridad por Rozada (1985). Como conclusión de su trabajo el autor señala que «... la Reforma... incorpora rasgos procedentes de los modelos antagónicos cientificistas y humanista... dualidad que... no nos parece por sí misma perniciosa... Pensamos que el error está en que no se recoge lo mejor que ofrecen cada uno de estos planteamientos... Puede decirse, creo, que la Reforma actual es cientificamentc subde. sarrollada y politicamente restrictivas. Cuando se haya finalizado el análisis de la concreción del modelo 
en el área de Ciencias Experimentales se podrá comprobar el grado de coincidencia entre los resultados que se obtengan y las conclusiones de Rozada.

\subsection{Los presupuestos didácticos de la Reforma y los objetivos comunes}

Los presupuestos didácticos aparecen por primera vez explícitamente en el documento Hacia la Reforma de 1985:

1. Atención preferente a la consecución de objetivos educativos que trasciendan el campo de las asignaturas.

2. Definición de una metodología activa.

3. Replanteamiento del sentido y el alcance de los contenidos.

4. Aproximación interdisciplinar entre las materias.

5. Revisión del sentido de la evaluación como instrumento de aprendizaje.

Los nueve objetivos comunes, tal y como aparecen en el mismo documento son:

1. Ser capaz de expresar oralmente y por escrito, de manera correcta y ordenada sus pensamientos y sentimientos.

2. Comprender los mensajes de la comunicación habitual.

3. Utilizar de forma crítica las fuentes de información.

4. Actuar de forma creativa.

5. Razonar con corrección lógica.

6. Tener una visión equilibrada e integrada de los distintos factores que conforman una realidad.

8. Tener un hábito racional de trabajo intelectual y manual y utilizar adecuadamente las técnicas respectivas.

9. Ser capaz de trabajar en equipo con un equilibrio entre la visión de conjunto y la tarea individual.

\subsection{Análisis de los objetivos}

Comenzará el análisis de las propuestas de la Reforma con el primero de los elementos del Modelo didáctico de Gimeno: los Objetivos.

En el proyecto de Reforma se atribuye una doble finalidad a las Ciencias Experimentales: "contribuir a la consecución de los objetivos comunes propuestos para el ciclo y capacitar a los alumnos para afrontar los problemas que le plantea su entorno físico con una actitud y una metodologia científicas" (Hacia la refor$m a, 1985)$. Estas intenciones educativas se concretan en los objetivos específicos del área: 19 objetivos de destrezas y habilidades científicas y 7 objetivos relativos a actitudes científicas. Los primeros se agrupan según se refieran:

1. Al dominio de la capacidad de observar

2. Al dominio de las técnicas de clasificación

3. A la familiarización con la formulación de hipótesis

4. A la iniciación a la experimentación
5. Al análisis de resultados y elaboración de conclusiones.

La formulación de estos objetivos parece basarse en taxonomías netamente psicológicas, las cuales permiten establecer objetivos en términos de funciones psicológicas, sin considerar el contenido científico de la disciplina en cuestion. Estas taxonomías (Gagné, Guilford, Bloom, D. Russell, Raths, citados por Gimeno, 1981) comparten el principio de la rindiferencia de los contenidos», según el cual los contenidos científicos de las distintas disciplinas son considerados como instrumentos para rellenar las funciones psicologicas, y son intercambiables, no importando demasiado qué contenidos se seleccionen.

Analizando estos objetivos desde otra óptica se puede deducir qué concepciones sobre el trabajo científico (o epistemológicas) los sustentan. Si bien es cierto que las destrezas y habilidades a las que se refieren los $19 \mathrm{ob}-$ jetivos son características del quehacer científico, no lo es menos que otros aspectos, a los que me referiré enseguida, hacen pensat en una visión inductivista y experimentalista de la Ciencia. En este sentido cabe senalar la importancia que se le concede a la observación - a la que en ocasiones se coloca en el punto de partida de una investigación-, la ausencia de referencias al planteamiento del problema objeto de estudio, $y$, fundamentalmente, la falta de reconocimeinto del papel que juega el marco conceptual de partida, no sólo en la observación sino en toda accion investigadora.

La presencia de los 7 objetivos actitudinales puede responder a una preocupación por la actitud negativa de la sociedad hacia la Ciencia en general y/o al convencimiento de que no se debe eludir, en un currículo de Ciencias, aspectos polémicos como el de relaciones Ciencia-Sociedad, la «neutralidad» de la Ciencia o su evolución histórica.

Para terminar con el análisis de los objetivos quiero referirme, aunque sea brevemente, a su nivel de abstracción. El grado de concreción de muchos de ellos y el afán de precisión en su formulación llevan a reconocer un enfoque conductista en su establecimiento. La pedagogía por objetivos que suele estar asociada se pone de manifiesto en los distintos elementos curriculares como veremos más adelante, fundamentalmente en la evaluación. La formulación de objetivos en términos de conductas observables o de las actividades que debe realizar el alumno en el aula, provoca en el profesorado cierta obsesión a la hora de diser̃ar las situa ciones de aprendizaje y al evaluar el grado de consecución de los objetivos, pues, tal como se nos dice, el alumno debe alcanzarlos todos al finalizar el cicto. Esta situación favorece, además, que el profesorado se cen. tre más en los detalles, en lo anecdótico, perdiendo de vista cuáles son realmente las finalidades que deben perseguirse. Así por ejemplo, en la práctica de los cuatro años de experimentacion, apenas se ha prestado atención a los objetivos actitudinales, no sólo ignorándo- 
los en ia evaluación, sino también, lo que es más preocupante, en la planificación y diseño de las programaciones.

No sólo la formulación de los objetivos hace pensar cn las semejanzas entre las propuestas de la Reforma y la pedagogia por objetivos, sino también, y principalmente, cl mismo papel que se atribuye a los objetivos en ellas:

- Los objetivos vienen ya determinados a un nivel de concreción tal que no es posible ningún grado de apertura con respecto a ellos, con lo que no se favorece el carácter abierto que debe tener el desarrollo curricular.

- Los objetivos se constituyen, junto con la evaluación, en el elemento curricular más importante, al que quedan supeditados los demás; son el punto de partida del proceso de desarrollo curricular que condiciona las posibles opciones que puedan tomarse en torno a los demás elementos curriculares.

En efecto, de los cinco presupuestos didácticos de la Reforma el primero es: "Atención preferente a la consecución de objetivos educativos que trasciendan el campo de las asignaturas". Estos objetivos educativos, concretados en los nueve objetivos comunes, se consideran "pieza clave» en el edificio de la Reforma; puntos de referencia para organizar y llevar adelante el proceso, constituyendo las materias medios para la obtención de los mismos; los cuales deben impregnar la pro. gramación y la metodología.

\subsection{Análisis de los contenidos}

En los presupuestos didácticos de la Reforma, como se ha visto, se considera preferente la atención que debe darse a los objetivos, considerados como «puntos de referencia para organizar y llevar adelante el proceso». Asimismo se ve la necesidad de replantear el sen. tido y el alcance de los contenidos y se declara que las materias "no constituyen fines en sí mismas; son me. dios para la obtención de dichos objetivos». Sc propone una selección de contenidos que conduzca a la sustitución de "contenidos inertes o cerrados" por otros relevantes, su intcgración y secuenciación acorde con la capacidad de los alumnos.

En el área de Ciencias Experimentales los contenidos sc presentan como un elemento curricular supeditado a los presupuestos básicos de la Reforma: la adquisición de objetivos comunes y de materia y el cambio me. todológico, si bien reconociendo que al alumno se lc debe proporcionar unos contenidos relevantes.

De estas declaraciones gencrales y de la consideración de la rapide $z$ con que en las últimas décadas se producen los conocimientos científicos, se deduce la necesidad primordial de abordar la selección de contenidos. Para cllo se proponen criterios de selección relacionados con las variables del sujeto que aprende y con los intereses sociales, haciéndose hincapié en su validez para alcanzar el mayor número de objetivos.
No resulta dificil concluir que las propuestas ministeriales se decantan hacia una conceptualización de los contenidos como medios o instrumentos para alcanzar objetivos educativos más amplios, constituyendo un ejemplo más de propuesta curricular que participa en el falso dilema objetivos-contenidos.

Con el fin de matizar más las opciones de la Reforma en torno al elemento contenidos, veremos qué tipos de contenidos se consideran, qué contenidos han sido seleccionados, como se han organizado y cuál es el grado de optatividad de los mismos.

En los documentos sobre la Reforma se habla de con* tenidos en un sentido bastante restrictivo, ya que atien. de sólo a los aspectos relacionados con la lógica inter. na de la disciplina, olvidando su significación psicopedagógica.

El concepto de contenidos es diferente, dentro del área de Ciencias Experimentales, scgún nos fijemos en las propuestas referentes a Ciencias Naturales o a Física y Química. Mientras en el primer caso se atiende a la significación psicopedagógica de los contenidos cientificos, además de a la lógica interna de la disciplina, en el caso de Física y Química la valoración y caracte. rización que se hace del conocimiento se centra en los aspectos lógicos, olvidando casi absolutamente los as. pectos psicológicos. Así, se incluye habitualmente en los contenidos los datos, hechos, conceptos, leyes, teo* rías, métodos, etc., acentuando más la atención en los procesos y métodos que en los conceptos y relaciones entre conceptos. No se hace referencia en los documentos de la Reforma a la construcción histórica del conocimiento científico ni a las interacciones CienciaSociedad, aspectos éstos de gran potencialidad motivadora.

Los contenidos seleccionados se organizan según dos opciones diferentes:

a) Ciencia integrada, distinguiéndose dos enfoques entre los que se experimentan: uno centrado en el entor. no y otro que se vertebra en torno a dos núcleos de interés: la materia y la energia.

b) Temario básico, por separado, de Ciencias Naturales y de Física y Química. La propuesta en Ciencias Naturales se desarrolla alrededor del tema del paisaje, mientras que en Física y Química el temario que se propone sigue la estructuración lógica de estas ciencias.

Debe señalarse que, a pesar de que en los documentos se declara que la programación debe orientarse hacia una interdisciplinaridad (no debe olvidarse que el cuarto de los presupuestos didácticos de la Reforma es la aproximación interdisciplinar entre las materias), a medida que el proceso experimental ha ido avanzando, son menos los centros que optan por la modalidad de Ciencia integrada, y algunos de los que inicialmente la eligieron, la han abandonado por las dificultades que supone su puesta en práctica. 
Puesto que he trabajado durante dos cursos como profesora de Física y Quimica en un centro experimental en el que se optó por el temario básico, me referiré algo más detalladamente a la selección de los contenidos y al grado de optatividad de esta opción.

Los contenidos seleccionados se han propuesto al profesorado experimentador de muy diferente forma a lo largo de los cuatro años. En el primer documento se hace una propuesta basada en la idea de cambio conceptual y metodológico acorde con el desarrollo historico que se concreta en cinco bloques:

1. La Mecánica como primera ciencia moderna.

2. Formas de interacciones fundamentales.

3. Origenes de la Quimica como ciencia moderna.

4. La Física y la Química a fines del siglo XIX.

5. La Química moderna como consecuencia de la nueva imagen del comportamiento de la materia.

Se hace hincapié en el carácter orientador y flexible de la propuesta para que el profesorado pueda variarla según las condiciones específicas en las que va a lievarla a la práctica.

En el segundo documento (publicado a los dos años de experimentación) se establecen unos «núcleos comunes a todas las opciones, que no pretenden cubrir la totalidad del tiempo del curso, sino garantizar una homogeneidad mínima entre todos los alumnos que finalicen el ciclo». En Física y Química estos contenidos básicos están estructurados en siete temas:

1. El movimiento.

2. Introducción a la Dinámica.

3. Calor. Energía.

4. Electricidad.

5. Estados de agregación y constitución de la materia.

6. Sistema periódico.

7. Reacciones químicas.

En cada uno de ellos se especifica con gran detalle el contenido a desarrollar y, puesto que se presentan como contenidos mínimos, deben incorporarse a todas las programaciones. Ahora el grado de libertad que se deja al experimentador consiste en organizarlos según la línea de Ciencia integrada o la del temario básico y constatar su viabilidad mediante la experimentación.

Como puede apreciarse, la tendencia en estos años se orienta hacia propuestas más cerradas y menos flexibles, que no consideran ni los aspectos psicopedagógicos de los contenidos científicos ni los procesos de construcción de la Ciencia a lo largo de la historia, ni las relaciones entre la Ciencia y la sociedad en la que se desarrolla.

Por último, una breve referencia al grado de optatividad de los contenidos. De la lectura del segundo documento puede inferirse que los contenidos propuestos representan un núcleo temático obligatorio que no debe ocupar la totalidad del tiempo dedicado a las Ciencias Experimentales en la distribución horaria del ci- clo. Sin embargo, en la práctica no sólo no es ésta la situación, sino más bien la contraria; es decir, la experiencia demuestra - al menos en los casos que conozco, con garantia de que se sigue una metodologia diferente a la transmisión de conocimientos ya elaborados- que el conjunto de contenidos seleccionados es demasiado extenso para que pueda desarrollarse en el Bachillerato General, al menos en las condiciones actuales. Es evidente, pues, que no puede hablarse de grado de opcionalidad en absoluto, lo cual no parece estar muy en consonaricia con un tratamiento integrador de la diversidad del alumno, asumido por la filosofía de la Reforma, según se deduce de la lectura de los documentos sobre evaluación.

\subsection{Relaciones de comunicación}

En los documentos analizados no aparece ninguna referencia explicita a los procesos de comunicación que se dan en el aula, ni orientaciones sobre cómo debe ser la estructura de la comunicación, su dirección, su contenido, quién ha de controlarla, qué recursos técnicos deben usarse como emisores, etc. Sin embargo, sí pueden deducirse algunas opciones respecto a este elemento a partir de declaraciones relacionadas con los fines de la Reforma, los objetivos comunes y las orientaciones didácticas.

Así, si se considera que con la Reforma se trata de «... fomentar... el trabajo en equipo" (Hacia la Reforma, 1985 , p. 5); si se tiene en cuenta que el objetivo común 9 es: «Ser capaz de trabajar en equipo con un equilibrio entre la visión de conjunto y la tarea individual» y que «se propugna la enseñanza activa, basada en la realización de actividades por el alumno» (Hacia la Reforma, 1985, p. 84), parece lógico suponer que se está optando por un modelo de comunicación caracterizado por favorecer situaciones en que exista más de un emisor y más de un receptor, la alternancia de roles entre amisor (es) y receptor (es), la comunicación «entre iguales» (alumno-alumno), un amplio espectro de contenidos de comunicación (informativos, de estructuración metodológica, de carácter personal-afectivo, etc.), la sustitución del emisor personal (habitualmente el profesor) por otros medios (libros, prensa, medios audiovisuales, etc.), cuando se trate de ciertos tipos de contenido de comunicación.

No puede darse por terminado el análisis de las propuestas de la Reforma respecto a las relaciones de comunicación sin referirse al profesor y a su función dentro del proceso de enseñanza-aprendizaje, ya que en el modelo didáctico elegido como instrumento para llevar a cabo este análisis, es precisamente en este elemento en el que se encuadra el papel del profesor.

En este sentido, la propuesta de una metodología activa atribuye al profesor la función de guía y orientador en el proceso de enser̃anza-aprendizaje, abandonando el rol de emisor por excelencia propio de estilos de enseñanza que favorecen la transmisión de conocimien- 
tos claborados. El profesor es, pues, un elemento más de la comunicación, que alterna los roles de emisor y receptor y da paso a la utilización de recursos técnicos como emisores en ciertos ámbitos del contenido comunicativo.

\subsection{La evaluación}

En los primeros documentos no se hace referencia explícita al tema de la evaluación, pero en el segundo «Libro verde» aparece como el quinto presupuesto didáctico de la Reforma la «revisión del sentido de la evaluación como instrumento de aprendizaje».

Han sido muchos los documentos, tanto generales como específicos del área de Ciencias Experimentales, aparecidos posteriormente, que han intentado clarificar la idea de evaluación que se asume en los planteamientos de la Reforma y orientar al profesorado en el proceso de evaluación.

En general, en todos ellos se insiste en que la evaluacion debe ser coherente con los principios de la Reforma y se opta por una evaluación formativa, dirigida al proceso de aprendizaje, continua y que atienda simultáneamente a la recuperación de los alumnos que la necesiten. Se trata de valorar no sólo el grado de consecución, por parte del alumno, de los objetivos comunes propuestos para el ciclo, sino también de contrastar la validez de las programaciones y metodologías de las diferentes áreas para conseguir dichos objetivos. Se intenta evaluar el progreso de cada alumno (por lo que se necesita una evaluación inicial que permita conocer el punto de partica de cada uno de ellos), evitando la "penalización» de los alumnos con menos «nivel». Cada profesor deberá revisar continuamente la programación de su materia, introduciendo las medidas correctoras oportunas dirigidas a superar las deficiencias que se manifiesten en la evaluación.

En lo que se refiere a las Ciencias Experimentales, en los documentos sobre evaluación se ha insistido, por una parte, en la correlación objetivos de materia/objetivos comunes, y por otra, en la elaboración y análisis de pruebas objetivas, con abundantes ejemplos ilustrativos. Sin embargo, poco o nada se dice sobre la recogida de información más continuada, necesaria para realizar una evaluación como la que se propugna, ni sobre los instrumentos que pueden emplearse, distintos de las pruebas escritas habituales.

El profesorado involucrado en estos años en la Reforma se ha encontrado con muchas dificultades de todo tipo, pero es quizás el tema de la evaluación el que ha suscitado más problemas. Los documentos enviados no han sido capaces de dar la información $y / o$ promover la reflexión y debate necesarios para su resolución. Después de cuatro años quedan sin responderse satisfactoriamente interrogantes importantes tales como: ¿Deben evaluarse los objetivos comunes o no? En caso afirmativo, ¿con qué frecuencia? ¿cómo: directamente o a través de los objetivos de materia? Y en caso negativo, ¿qué evaluar: los objetivos de materia, los contenidos de las distintas disciplinas?

Y si el profesorado ha encontrado grandes dificultades en la evaluación y calificación de los alumnos, la evaluación de las programaciones, las metodologías seguidas y las propias actuaciones de los profesores ha sido, en general, desatendida en la práctica. Es en este terreno, quizás, donde más se evidencia el modelo de profesor que abunda, poco habituado a la reflexión critica, individual o colectiva, de su propia práctica, y con pocos recursos para abordar un proceso de investigación-acción como el que se propone. Mientras esta situación no sea tenida en cuenta y se integren en la política de formación del profesorado medidas en. caminadas a modificarla, muy poca utilidad tendrán todos los documentos que puedan elaborarse sobre ei tema; al menos la experiencia de estos años así lo demuestra.

\subsection{Organización}

Dadas las caracteristicas de este trabajo sólo me referiré a algunas de las variables de organización que más directamente se relacionan con las propuestas de la Reforma en el área de Ciencias Experimentales.

Una de ellas es el horario, que establece que de las 30 horas lectivas en cada curso, a las Ciencias Experimentales se dediquen 4 horas en primero y 3 horas en segundo; y, como lo que se está generalizando es dar en primero las Ciencias Naturales y la Física y Química en segundo, a esta asignatura le corresponden, pues, 3 horas semanales en este ciclo. Como se serialaba al analizar los contenidos, las tres horas resultan insuficientes si se quiere desarrollar, con una metodología activa, el conjunto de contenidos básicos seleccionados.

Otro de los aspectos relacionados con la organización es el agrupamiento de los alumnos. Aunque no se dice nada explicitamente, sí puede deducirse que se propo. ne fomentar el trabajo en equipo de los alumnos, y por tanto, organizarlos en pequeños grupos, principalmente para la ejecución de ciertas tareas tales como pequenaas investigaciones, preparación de trabajos que requieran consultas bibliográficas, etc.

También puede considerarse en este elemento la organización de los espacios. La utilización del laboratorio según el tipo de actividad que tengan que realizar los alumnos; un aula que permita organizar a los alum. nos en pequeños grupos o dispuestos individualmente, que disponga de una biblioteca que puedan usar los alumnos en cualquier momento, con paneles adecuados para la exposición de trabajos, noticias de la prensa, etc., son ejemplos de otros aspectos organizativos que se desprenden de los planteamientos psicodidácticos de la Reforma.

En la práctica, muchas de estas condiciones no se dan 
y muchos planteamientos didácticos se tienen que sacrificar.

\subsection{Metodología}

En el modelo didáctico propuesto por Gimeno no se considera el método como un elemento más del modelo con entidad propia. En este contexto el método es considerado como la «síntesis práctica de opciones tomadas en variables de orden psicológico, didáctico y filosófico, que en muy buena medida se agotan en las dimensiones detectadas en los elementos del modelo didáctico que hemos desarrollado»... «Es decir, que para analizar un método hay que acudir a dimensiones de otros elementos didácticos» (Gimeno, 1981, p. 227).

Esto es lo que he hecho hasta aqui: determinar las opciones que se han tomado en la Reforma frente a las dimensiones de los elementos del modelo didáctico para configurar cuál es el modelo metodológico que se propugna y, por un proceso de inferencia, deducir cuáles son los principios psicodidácticos básicos que lo sustentan, esbozando, por último, el modelo de enseñanzaaprendizaje de las Ciencias que subyace en esos planteamientos.

Aunque ya se ha ultimado esta primera tarea (determinación de las opciones tomadas frente a los elementos del modelo didáctico), creo que para tener una idea más clara del modelo metodológico que se propone, debo analizar el contenido de los documentos que se refieren explícitamente a metodología.

En los documentos de la Reforma se afirma que «una buena metodología es, en útimo término, cualquier procedimiento eficaz para conseguir los objetivos propuestos», y en la introducción ya me he referido a los Presupuestos didácticos de la Reforma, el segundo de los cuales es "Definición de una metodología activa".

La concreción de estas ideas generales en el área de las Ciencias Experimentales se hace ofreciendo dos modalidades: aproximación a una metodología multidisciplinar, basada en una visión conjunta del medio físico y en el empleo de una metodología común en las diferentes ciencias; y un método activo, basado en la realización de actividades por el alumno, a fin de que éste adquiera un método de trabajo y unas capacidades que, con unos contenidos relevantes, le permitan progresar en la consecución de los objetivos. Estas actividades serán semejantes a las desarrolladas por los científicos en sus trabajos de investigación. Se aclara que la defensa del método activo no supone una propuesta de «activismo» (aunque en la práctica es to que ha ocurrido muchas veces) y se propugna un aprendizaje científico significativo.

En posteriores documentos se han desarrollado aún más estas ideas, con el propósito de orientar al profesorado en la organización y desarrollo de sus clases. En el documento sobre programación (8) se afirma que, puesto que no existe en la actualidad una teoria del aprendizaje válida para la adolescencia, tampoco hay una teoría de la instrucción y, por tanto, puede haber muchas alternativas válidas para el intento de uracionalizar» el proceso de enseñanza-aprendizaje. Se concluye diciendo que el modelo idóneo es el que: a) funcione más o menos bien, $\mathrm{y}$ b) el que permita a profesores y alumnos trabajar a gusto.

Más adelante se centra dicho documento en las estrategias de ensenanza, concebidas éstas como las actividades correspondientes a períodos de tiempo más cortos y dirigidos a la consecución de unos objetivos operativos concretos. Se proponen criterios para la selección de actividades en el caso de que más de una sean adecuadas para un tipo de objetivo dado.

Como comentario a esta sintesis de las propuestas metodológicas, me quiero referir a la propia denominación de método activo. El témino en sí es lo suficientemente amplio y ambiguo como para poder incluir en él cualquier modelo metodológico que dé importancia a la actividad del alumno en el aula, aunque se basen en ideas diferentes sobre el proceso de aprendizaje. Así, la enseñanza por descubrimiento, los modelos basados en una visión constructivista del aprendizaje y hasta los que se fundamentan en la teoría del aprendizaje por transmisión verbal, a pesar de sus diferencias, pueden considerarse como ejemplos de métodos activos.

Es cierto que la propuesta de método activo se ha concretado en la idea de que el alumno realice en el aula actividades análogas a las desarrolladas por los científicos en sus investigaciones, o lo que es lo mismo, se pretende que el alumno se familiarice con la metodología científica. En este sentido, puede antadirse que esta propuesta se extiende a todas las disciplinas, concibiendo de esta manera la interdisciplinaridad metodológica (11). Ahora bien, en ninguno de los documentos específicos del área de Ciencias Experimetales se hace referencia a las investigaciones en didáctica de las Ciencias, muchas de las cuales han señalado las deficiencias de algunos planteamientos que persiguen la adquisición de la metodología científica por parte del alumno, debido, en cierta medida, a la concepción del trabajo cientifico que subyace en ellos (Gil, 1983).

\subsection{EI modelo subyacente}

Después del análisis realizado resulta dificil relacionar las propuestas de la Reforma con alguno de los modelos de enseñanza-aprendizaje de las Ciencias que en la actualidad orientan su enseñanza. Y esto es así debido a que en estas propuestas pueden encontrarse rasgos propios de más de uno. Procederé, pues, a establecer las características que configuran el modelo de la Reforma.

La consideración que se hace de los objetivos - tanto de los comunes como de los de materia -está en la linea de los planteamientos de la pedagogía por objetivos y la psicología conductista en que se apoya, como 
he tenido ocasión de mostrar al analizar este elemento: objetivos expresados en términos de conductas observables, preocupación por la precisión en su formulación, preeminencia de los objetivos frente al resto de los elementos curriculares, evaluación del grado de consecución de los mismos.

La concepción que se asume de la Ciencia y de la forma en que se produce el conocimiento científico parece estar más próxima a las concepciones inductivistas y empiricistas. Si bien esto es algo que no aparece de forma explícita en los documentos, sí puede deducirse del propio contenido de los objetivos de materia, del papel que se le atribuye a los contenidos científicos, de las orientaciones didácticas y de los ejemplos de unidades didácticas que se han enviado a los profesores como material de apoyo.

No se hace referencia a ninguna teoría del aprendizaje en particular, ni siquiera a la idea de aprendizaje que se asume; más bien se señala la ausencia de una teoria válida para explicar como se aprende y de la que puedan obtenerse prescripciones didácticas bien fundamentadas, y se remite al profesorado al "sentido común" como único bagaje teórico para la reflexión en profundidad que requiere la organización y desarrollo de su práctica. Estas recomendaciones tan peligrosas y tendenciosas (i iqué concepto de profesor se corresponde con ellas? ?) pueden producir en el profesorado, si no desorientación, sí despreocupación por el conocimiento de otras disciplinas estrechamente relacionadas con la enseñanza, y ante las cuales existe un tradicional rechazo.

Las orientaciones metodológicas apuntan más bien hacia un aprendizaje por descubrimento, con la visión inductivista y experimentalista del quehacer cientifíco que subyace en este modelo y al que ya me referi antes; $y$ aunque se declara la necesidad de perseguir un aprendizaje científico significativo, no se profundiza en el contenido de esta expresión ni se remite a ninguna teoría psicológica que lo haya definido. También se comparte con el modelo de aprendizaje por descubrimiento la preponderancia de los procesos y métodos de la Ciencia sobre el conocimiento científico, poniendo más énfasis en el desarrollo de destrezas y habilidades cientificas (objetivos de materia) por parte del alumno que en la comprensión y aprendizaje significativo de los conceptos básicos. Y para terminar con el paralelismo que observo entre las propuestas de la Reforma y el aprendizaje por descubrimiento, señalar la poca importancia que se le da al marco conceptual del alumno con el que se va a enfrentar a las experiencias de aprendizaje de las Ciencias que vivirá en el aula.

En efecto, y enlazándolo con lo anterior, resulta verdaderamente sorprendente que, siendo la investigacion de las ideas previas de los alumnos sobre conceptos científicos uno de los campos más estudiados en los últimos antos en la didáctica de las Ciencias - como se verá en la segunda parte de este trabajo- apenas se haga referencia a ello en una propuesta que pretende modificar significativamente la enseñanza de las Ciencias.

Y no sólo en este campo no se hace referencia a la investigación didáctica, sino que en general, es la gran ausente en los documentos sobre la Reforma; no ya porque no existan referencias explícitas a ella (se habla de la ausencia de una teoría del aprendizaje suficientemente contrastada pero no se dice nada de la investigación didáctica, más próxima al quehacer del aula) sino porque se siguen defendiendo planteamientos cuya ineficacia y deficiencias han sido reiteradamente evidenciadas por la investigación didáctica.

Otra propiedad atribuible al modelo de la Reforma es su carácter cerrado e inflexible. Al profesor experimentador le quedan pocos grados de libertad a la hora de planificar el curso: con unos objetivos de materia que ya vienen dados y muy especificados, con unos contenidos "mínimos» que no son tal y poseen un elevado grado de concreción, al profesor sólo le queda la posibilidad de diseñar unas actividades que cumplan la condición de poner en práctica las destrezas a las que se refieren los objetivos, utilizando los contenidos propuestos.

Otra manifestación del carácter cerrado del modelo es la ausencia de un diseño de investigación que contemple la evaluación interna del proceso de enseñanzaaprendizaje, y que permita al profesor realizar la investigación-acción que se le reclama. Sí se encuentran con gran profusión en los documentos sobre la evaIuación declaraciones sobre la necesidad de concebir ésta no sólo como una evaluación del alumno sino también del proceso, y como instrumento para comprobar la validez de las programaciones y las metodologías empleadas. Sin embargo, no se ofrece al profesorado un diseño minimo, ni siquiera unas orientaciones generales de cómo Ilevar a cabo esa evaluación, qué instrumentos y técricas puede emplear; y eso es indispensable si se tiene en cuenta que el profesorado, en general, no está acostumbrado a la reflexión sistemática y organizada sobre su práctica, y que las modificaciones e innovaciones que emprende, caso de hacerlo, en la gran mayoría de las ocasiones estân fundamentadas en la intuición y la improvisación.

Quería referirme al papel del profesor en el modelo, a la concepción que se tiene de su función, y en parte ya he comenzado a hacerlo en el párrafo anterior. Conviene subrayar que en las propuestas de la Reforma se atribuye un importante protagonismo al profesorado en todo el proceso; sin embargo, tal como afirma Rozada (1985), si se intenta generalizar por decreto un curriculo, aunque se presente avalado por una experimentación en la que haya participado un cierto número de profesores, sólo habrán ejerciclo ese protagonismo esos profesores que hayan participado en la experimentacion y hayan estado además de acuerdo con su desarrollo y conclusiones. 
En resumen: el modelo de enserianza-aprendizaje de las Ciencias que subyace a las propuestas de la Reforma, comparte características de la pedagogia por objetivos y la psicología conductista, así como del modelo de aprendizaje por descubrimiento; el diseño curricular en el que se concreta es de carácter cerrado, quedando muy limitada la participación del profesorado. Se acusa una falta de fundamentación teórica de las propuestas, tanto en el ámbito de la Psicología como en el de la Epistemología.

\section{LA INVESTIGACIÓN DIDÁCTICA Y EL DESARROLLO CURRICULAR}

No es mi intención hacer una revisión de los problemas más importantes abordados durante los últimos años en la investigación didáctica; tampoco haré una relación de las aportaciones más significativas de esta investigación al desarrollo curricular, puesto que todo ello sobrepasaría los límites de este trabajo.

Sin embargo, me parece oportuno señalar las características comunes a los diferentes modelos de enseñan $\mathrm{za} /$ aprendizaje de las Ciencias propuestos recientemente (Posner et al., 1982; Gil y Carrascosa, 1985; Osbor * ne y Wittrock, 1985; Driver, 1986), y referirme principalmente a las implicaciones que para el desarrollo del currículo pueden extraerse de estos modelos.

\subsection{Las ideas de los estudiantes}

Actualmente parece existir un acuerdo mayoritario en la necesidad de considerar las concepciones, creencias, hábitos y expectativas de los alumnos como punto de partida obligado en la construcción del currículo. De ahí la importancia que tiene que profesores y alumnos dediquen tiempo y esfuerzo a conocerlos, valorarlos y estudiar las formas de modificarlos.

\subsection{El aprendizaje como cambio conceptual, metodo- lógico $\mathbf{y}$ actitudinal}

Si bien debemos reconocer que no existe un cuerpo de conocimientos coherente y suficientemente contrastado que de cuenta del complejo proceso de aprendizaje, si se puede diferenciar las concepciones del aprendizaje que han venido mostrando su ineficacia de las que parecen más prometedoras y con más potenciali. dad explicativa. El enfoque constructivista del aprendizaje y, en particular, la concepción de éste como cambio conceptual es un supuesto compartido básicamente por los modelos de enseñanza-aprendizaje a los que me he referido antes. Parece abrirse paso la idea más amplia de aprendizaje como cambio conceptual, metodobogico y actitudinal, que de ser asumida por quienes construyen y desarrollan el currículo, debería llevar a cambios profundos en el trabajo del aula. Adoptar esta visión del aprendizaje supondrá, entre otras cosas:

a) El diseño de situaciones de aprendizaje y la utiliza- ción de recursos adecuados para favorecer el conocimiento de las condiciones iniciales de los alumnos, provocar el conflicto cognitivo que produzca en éstos insatisfacción con sus ideas, favorecer la construcción de concepciones alternativas que les resulten comprensibles y más útiles que las iniciales, y darles oportunidades para que utilicen las nuevas ideas y comprueben sus posibilidades.

b) Introducir en la clase de Ciencias la metodologia científica, procurando que los alumnos se familiaricen con ella utilizándola en todas las ocasiones posibles. No debe olvidarse que muchos modelos que han pretendido familiarizar a los alumnos con el método cientifico lo que han conseguido ha sido desvirtuar el trabajo científico y dar a los estudiantes una imagen falseada de la Ciencia. Hay que insistir en aquellos aspectos del trabajo científico más desatendidos en las aulas y que quizás sean los más caracteristicos del quehacer científico: los relacionados con la creatividad propia del pensamiento divergente (emisión de hipótesis, diseño de experiencias) y la contrastación en condiciones controladas de las hipótesis de partida.

c) La utilización de la Historia y la Epistemologia de la Ciencia como recurso que no solamente facilitará el cambio conceptual y metodológico sino que favorecerá el cambio actitudinal de los alumnos hacia la Ciencia y su aprendizaje, al mostrar a éstos los aspectos más controvertidos y polémicos del desarrollo científico en diferentes momentos historicos y contextos socioculturales, sin olvidar los actuales.

d) El conocimiento de las relaciones entre las diferentes ciencias, entre éstas y la tecnología y otras disciplinas, acrecentará el cambio actitudinal y la motivación de los alumnos hacia el aprendizaje, condición necesaria para que éstos adopten una actitud activa en el proceso de enseñanza-aprendizaje, y se produzcan por tanto los cambios buscados.

\subsection{Concepción del currículo}

Una consecuencia de la visión del aprendizaje que se ha expuesto es que el currículo no puede concebirse como un proyecto diseñado de antemano por personas más o menos ajenas a la realidad del aula. La idea de currículo que me parece más coherente con el modelo de enseñanza-aprendizaje que se propugna, consiste en considerarlo como la «práctica consciente del profesorado centrada en la elaboración de estrategias de enseñanza mediante la toma de decisiones en los elementos que la configuran y sobre las que se puede incidir personal o colectivamente" (Arrieta, Cascante y Rozada, 1987, p. 26).

Esta concepción implica el diseño de situaciones y materiales de aprendizaje a partir de decisiones tomadas por el profesor, individual $y / o$ colectivamente, con el asesoramiento de expertos que se necesite, y fundamentándose tanto en las teorias de las disciplinas relacio- 
nadas con la educación, como en su propia experiencia práctica.

\subsection{Papel del profesor}

Una nueva idea sobre el currículo y su desarrollo implica necesariamente una concepción diferente de la función del profesor. El punto de vista sobrc el currículo que he expuesto antes atribuye al profesor un papel distinto al del transmisor neutral de conocimientos sobre los que se le supone más o menos experto. El profesor se concibe más bien como "colaborador de investigación en el desarrollo, implementación y evaluación de materiales y métodos didácticos» (Driver, 1986, p. 111). Yo prefiero atribuirle una tarea más amplia y ver al profesor como un «investigador curricular que trata de aclarar hechos para ir transformando su propia forma de trabajo, y que, al mismo tiempo que hace esto, accede a niveles superiores de pensamiento general, que se traduce en nuevas pautas de actuación para la innovación curricular" (Arrieta, Cascante y Rozada, 1987, p. 48).

\subsection{El trabajo de los alumnos}

Existe la opinión generalizada entre la mayoría de los investigadores en didáctica de que el trabajo en equipo, el intercambio de ideas, las discusiones, favorecen los cambios conceptuales, metodológicos y actitudinales que conllevat el aprendizaje. Esto tiene repercusiones en la organización de la clase y del trabajo que en ella se desarrolle. Cualesquiera que sean las decisiones que se tomen al resnecto, lo que debe procurarse es of recer a todos los alumnos oportunidades reiteradas para hacer explícitas sus ideas, comunicarlas, contrastarlas y modificarlas si es preciso.

\section{HACIA LA RFFORMA DE LA ENSEÑAN- ZA DE LAS CIENCIAS}

Una vez analizadas las propuestas de la Reforma en cl área de Ciencias Experimentales y teniendo en cuenta las aportaciones de la investigación didáctica de las Ciencias al desarrollo curricular, parece oportuno senalar aquellos aspectos que, a mi entender, deben ser tenidos en cuenta si se quiere abordar una profunda innovación de la enseñanza de las Ciencias, y que, quizás, sean los más desatendidos en el proyecto de la Reforma.

a) Las propuestas oficiales deben explicitar cuáles son las concepciones que se sustentan sobre la educacion en general y la educación escolar en particular, el conocimiento, cl aprendizaje, la Ciencia y su desarrollo, el currículo, el profesor,...

b) Las innovaciones que se proyecten y lieven a la práctica deben estar conectadas con la investigacion en doble sentido: utilizando sus resuitados y las implicaciones pedagogicas que de ellos se derivan, y desembocan- do en investigación. Sólo asi puede esperarse que la enseñanza de las Ciencias esté en el futuro menos expuesta a los vaivenes de las últimas décadas.

c) Las propuestas deben ser lo suficientemente abiertas y flexibles para que el profesorado pueda realizar esa labor de investigación que se deduce del párrafo anterior, y para que aquéllas puedan adaptarse a las condiciones concretas en las que van a ser llevadas a cabo.

d) Las implicaciones que para ei desarrollo curricular se pueden deducir de la investigación didáctica y que he intentado resumir en el punto 3, dcben ser incorporadas ya a las propuestas innovadoras, considerán. dolas como hipótesis de partida, susceptibles de ser modificadas o sustituidas por otras. En particular, deben rechazarse las propuestas que estén relacionadas con modelos de enseñanza-aprendizaje que han mostrado sobradamente su ineficacia y deficiencias (aprendizaje por descubrimiento, Ciencia integrada, enseñanza por transmisión).

e) Debe darse más importancia a la evaluación inter. na de las propuestas, incluyendo un diseño de investigación como instrumento que, en manos del profesor, le permita realizar la investigación-acción que se suponc debe abordar.

f) Es impensable una reforma de la enseñanza de las Ciencias sin participación activa del profesor, pero, fundamentalmente, sin un cambio en profundidad del pensamiento del profesorado. Este cambio, que implica, como señala Gil (1985), un cambio metodológico, actitudinal y diđáctico, es, en esencia, el perfeccionamiento del profesorado que rcalmente se necesita, y quc es indisociabie de la innovación y desarrollo curriculares. Es decir, como afirman Arrieta, Cascante y Rozada (1987, p. 48), «la investigación, el desarrollo, la innovación, el perfeccionamiento e incluso la formación inicial, no son más que diferentes caras de un mismo cuerpo, de una misma actividad, que empieza a distorsionarse en el momento que se producen sin tener en cuenta que son un todo indisociable».

\section{CONCLUSIONES}

El estudio de los documentos que recogen las propuestas oficiales del MEC para la Reforma de las Enseñanzas Medias, y cn especial las que se refieren al área de Ciencias Experimentales, ha permitido la caractcrización del modelo de enseñanza-aprendizaje de las Cicncias que subyace en tas mismas y que no aparece en ellas de forma explicita. Como resultado del análisis se subrayan los planteamientos que comparten estas propuestas con la pedagogía por objetivos y el modelo de aprendizaje por descubrimiento.

La revisión de las implicaciones para el desarrollo curricular de la investigación didáctica reciente se toma como referencia para señalar qué aspectos de las pro- 
puestas deben ser modificados y, en definitiva, qué requisitos debe cumplir cualquier proyecto innovador de la enseñanza de las Ciencias que pretenda transformar significativamente la situación actual.

\section{REFERENCIAS BIBLIOGRÁFICAS}

ARRIETA, J., CASCANTE, C. y ROZADA, J.M., 1987, Bases para el desarrollo curricular (Primer borrador), Oviedo.

AUSUBEL, D.P., 1978, Psicologia educativa. Un punto de vista cognoscitivo, (Trillas: México).

BLACK, P., 1986, Presidential address: Integrated or coordinated Science?, The School Science Review, vol. 67, pp. 669-681.

CARRASCOSA, I., 1985, Errores conceptuales en la ensenanza de la Física y la Quimica: una revisión bibliográfica, Ensenanza de las Ciencias, vol. 3, pp. 230-234.

CARRASCOSA, J. y GIL, D., 1985, La "metodologia de la superficialitat» i l'aprenentatge de les ciències, Enseñanza de las Ciencias, vol. 3, pp. 113-120.

DRIVER, R., 1986, Psicología cognoscitiva y esquemas conceptuales de los alumnos, Enseñanza de las Ciencias, vol. 4, pp. 3-15.

DRIVER, R. y ERIKSON, L., 1983, Theories into action: some theoretical and empirical issues in the study of students' conceptual frameworks in science, Studies in Science Education, vol, 10, pp. 37-70.

DRIVER, R. y OLDHAM, V., 1986, A constructivist approach to curriculum development in Science, Studies in Science Education, vol. 13, pp. 105-122.

GIL, D., 1982, La investigación en el aula de Física y Quimica, (Anaya: Madrid).

GL, D., 1983, Tres paradigmas básicos en la enseñanza de las Ciencias, Enseñanza de las Ciencias, vol. 1, pp. 26-33.
GIL, D., 1985, El futuro de la ensetanza de las Ciencias: algunas implicaciones de la investigación educativa, Revisto de Educación, n 278 , pp. 27-38.

GIL, D., 1986a, Bases teóricas de un modelo de ensehanza. aprendizaje de las Ciencias, Simposio Psicología del Aprendizaje y Desarrollo Curricular, Oviedo.

GIL, D., 1986b, La metodología científica y la enseñanza de las Ciencias: unas relaciones controvertidas, Enseftanza de las Ciencias, vol. 4, pp. 111-121.

GIL, D. y CARRASCOSA, J., 1985, Science learning as a conceptual and methodological change, European Journal of Science Education, vol, 7, pp. 231.236.

GIL, D. y MARTINEZ-TORREGROSA, J, 1983, A model for problem solving in accordance with scientific methodology, European Journal of Science Education, vol. 5 , pp. 447.455 .

GIMENO SACRISTAN, J, 1981, Teoría de la enseñanza $y$ desarrollo del currículo, (Anaya: Madrid).

GUTIERREZ, R., 1987, Psicologia y aprendizaje de las Ciencias: el modelo de Ausubel, Enseñanzo de las Ciencias, vol. 5, pp. 118-128.

HASHWEH, M.Z., 1986, Toward an explanation of conceptual change, European Journal of Science Education, vol. 8, pp. 229-249.

HODSON, D., 1985, Philosophy of Science, Science and Science education, Studies in Science Educalion, vol. 12 . pp. 25-57. 
MATA, A. y ANTA, C., 1985, Evolución y nuevas tendencias en los trabajos sobre didáctica de las Ciencias Experimentales. Revisión del año 1984, Enseñanza de las Ciencias, vol. 3, pp. 167-172.

MATA, A. y MENDEZ, A., 1985, La renovación didáctica dc las Ciencias Experimentales: estudio bibliométrico, Ensehanza de las Ciencias, vol. 3, pp. 3-10.

McCl.ELLAND, J.A.G., 1984, Alternative frameworks: interpretation of evidence, European Journal of Science Education, vol. 6, pp. 1-6.

McDERMONT; L.C., 1984, Critical review of research in the domain of Mechanics, Research on Physics Education, (Editions du CNRS: Paris).

MILLAR, R. y DRIVER, R., 1987, Beyond processes, Studies in Science Education, 14, 33-62.

MINISTERIO DE EDUCACION Y CIENCIA, 1987, Proyecto para la reforma de la enseñanza. Educación infantil, primaria, secundaria y profesional, Madriđ.

NOVAK, J.D., 1982, Teoria y práctica de la educación, (Alianza Universidad: Madrid).

OSBORNE, R. y WITTROCK, M., 1983, Learning Science: a generative process, Science Education, vol. 67, pp. 450.508 .

OSBORNE, R. y WITTROCK, M., 1985, The Gencrative Learning Model and its implications for Science education. Studies in Science Education, vol. 12, pp. 59-87.

POSNER, G.J., STRLKE, K.A., HEWSON, P.W. y GERT ZOG, W.A., 1982, Accomodation of a scientific conception: towards a theory of conceptual change, Science Education, vol. 66, pp. 211-227.
ROZADA, J.M., 1985, Análisis del modelo didóctico de la Reforma, (Centro de Profesores: Gijón).

YAGER, R.E. y PENICK, J.E., 1983, Analysis of the curent problems with school Science in the USA, European Journal of Science Education, vol. 5, pp. 459-463.

\section{ANEXO}

Documentos del MEC sobre la Reforma de EEMM examinados para este trabajo:

1. Hacia la Reforma. Documentos, 1983.

2. Hacia la Reforma. Documentos, 1985.

3. Hacia la Reforma, Documentos complementarios I, 1985

4. Hacia la Reforma. Documentos complementarios II, 1986.

5. Prueba de evaluación de objetivos especificos, 1985 .

6. Algunos datos sobre prucbas de evaluación y su análisis, 1985.

7. Ponencia para la revisión del Libro Verde, 1985.

8. Ideas generales sobre programación, 1986.

9. La evaluación en Ciencias Experimentales, 1986.

10. Formulación operativa de contenidos básicos de Fisica y Quimica, 1986.

11. Interdisciplinaridad, 1986. 